\title{
CONSEQUÊNCIAS DO USO ABUSIVO DE SUBSTÂNCIAS PSICOATIVAS POR PESSOAS EM TRATAMENTO
}

\section{Fernanda Carolina Capistrano}

Doutoranda do Programa de Pós-graduação em Enfermagem da UFPR.

\section{Gustavo Jorge Maftum}

Médico residente em pediatria, Brasil.

\section{Maria de Fátima Mantovani}

Doutora em Enfermagem, Docente do PPGENF/UFPR. Coordenadora do Grupo de Estudos Multiprofissional em Saúde do Adulto - GEMSA, Brasil.

\section{Jorge Vinícius Cestari Felix}

Doutor em Ciências, Docente do PPGENF/UFPR Membro do Grupo de Pesquisa e Inovação em Saúde, Brasil.

\section{Luciana Puchalski Kalinke}

Doutora em Ciências da Saúde. Docente do PPGENF/ UFPR. Membro do GEMSA. Editora da Revista Cogitare Enfermagem, Brasil.

\section{Miriam Aparecida Nimtz}

Doutora em Enfermagem. Professora Adjunta do Departamento de Enfermagem da UFPR. Universidade Federal do Paraná, Curitiba (PR), Brasil. Membro do NEPECHE.

\section{Mariluci Alves Maftum}

Doutora em Enfermagem. Docente do PPGENF/UFPR. Vice coordenadora do NEPECHE.
Autor correspondente: Fernanda Carolina Capistrano E-mail: fernanda_capistrano@yahoo.com.br
RESUMO: Este artigo tem como objetivo caracterizar o impacto do uso abusivo de substâncias psicoativas para a pessoa com transtorno relacionado a substâncias. Trata-se de um estudo quantitativo, analítico e transversal, com 163 pacientes de um Centro de Atenção Psicossocial Álcool e Drogas. Os dados foram coletados com a aplicação individual de um instrumento estruturado e analisados por meio descritivo e estatístico com teste de correlação de Person, Teste de Média e Qui-quadrado. Como resultado identificou-se que a maioria são homens, que utilizam álcool e crack, baixa escolaridade, solteiro, desempregado e baixa renda. Houve abandono escolar, problemas laborais e mudança de ocupação. Tiveram episódios de overdoses, suicídios, comorbidades mentais, físicas, comportamento sexual de risco, Doenças Sexualmente Transmissíveis (DST's), agressões físicas, crimes e acidentes de trânsito. Foi identificado histórico do uso de drogas, rompimento de vínculos, separação, abandono do lar e situação de rua. Conclui-se que o uso abusivo de drogas afeta as pessoas direta e indiretamente em todos os âmbitos da vida, pessoal, laboral e acadêmico e social.

PALAVRAS-CHAVE: Transtornos relacionados ao uso de substâncias; Saúde mental; Enfermagem psiquiátrica.

\section{CONSEQUENCES OF ABUSE FROM PSYCHOACTIVE SUBSTANCES BY PEOPLE UNDER TREATMENT}

\begin{abstract}
The impact of psychoactive substances on the person with disorders related to illegal drugs is analyzed. Current quantitative, analytic and transversal study comprised 163 patients from an Alcohol and Drug First Aid Center. Data were retrieved by applying a structured tool to each individual and analyzed descriptively and statistically by Pearson's correlation test and by mean and Chi-square tests. Results show that most people who take alcohol and crack have low schooling level, they are single, unemployed and have a low income. Schooling was forsaken at an early age and work problems and occupation changes occurred, coupled to overdose episodes, suicide attempts, mental and physical co-morbidity, risky sexual behavior, sexually transmitted diseases (STDs), physical aggressions, crimes and street traffic accidents. A history of drug use, breaking of family bonds, separation, abandonment of home and street living was constant. Results show that the abuse of drugs affects people directly and indirectly with regard to their personal, labor, academic and social life.
\end{abstract}

KEY WORDS: Substance-Related Disorders; Mental Health; Psychiatric Nursing. 


\section{INTRODUÇão}

Desde meados do século $\mathrm{XX}$, os transtornos relacionados a substâncias tornou-se grave problema mundial de saúde pública com emersão de vasto impacto biológico, psicológico, econômico e social. Representa um dos maiores desafios a ser enfrentado em decorrência do crescente número de casos e da alta complexidade de fatores que envolvem esse transtorno ${ }^{1.3}$.

Neste complexo universo, o uso da substância química, por si só, é um dos fatores presentes no desenvolvimento da síndrome de dependência. Somam-se a este outros como o próprio indivíduo e a sociedade, fundamentais para a ocorrência desse transtorno mental e, por consequência, o surgimento de impactos negativos em diferentes âmbitos da vida ${ }^{1-3}$. Destarte, concentrar-se somente no uso de substâncias é minimizar todos os elementos importantes envolvidos no uso abusivo e na dependência, isso porque representa um aspecto dentre o amplo conjunto de fatores envolvidos.

Sabe-se que ao menos 15,3 milhões de pessoas no mundo possui transtornos relacionados a substâncias, incluindo o álcool e outras drogas ${ }^{4}$. Somente no ano de 2017, cerca de 250 milhões de pessoas, correspondente a $5 \%$ da população mundial adulta, utilizaram algum tipo de droga sem considerar as drogas lícitas e, desses, 29,5 milhões são considerados consumidores problemáticos. ${ }^{5}$

O início do uso de substâncias psicoativas geralmente acontece na adolescência, púberes tendem a iniciar em média aos 12,5 anos com o consumo de bebidas alcoólicas e tabaco, substâncias liberadas para a produção, venda e consumo no Brasil ${ }^{1,6}$. Já as substâncias ilícitas, a média de início do consumo sobe para 13,9 anos para a maconha e 14,4 anos para a cocaína. Este panorama nacional difere do encontrado na década de 1950, em que o início de envolvimento com as substâncias psicoativas ocorria em torno dos 18 anos de idade ${ }^{1,6}$.

Diante desse cenário, compreende-se que o consumo precoce de drogas contribui com o desenvolvimento de comportamentos delinquentes, elevam os índices de mortalidade prematura, favorece um comportamento sexual de risco, insucessos escolares e laborais, perpetração ou vitimização de comportamento violento, além disso pode aumentar o risco de desenvolver comorbidades mentais como a depressão e ansiedade ${ }^{7}$.
O impacto dos transtornos relacionados a substâncias é incalculável, pois reduz as condições e a qualidade de vida atingindo intensamente a sociedade como um todo. É um dos transtornos mais comuns da humanidade, ordinária a várias pessoas, independente da etnia, religião, gênero ou condição social ${ }^{3,7}$.

Este artigo se justifica pela importância e pela necessidade de o enfermeiro e a equipe multiprofissional que trabalham com pessoas com transtornos relacionados a substâncias, conhecer os fatores envolvidos nesse universo complexo, multivariado e dinâmico e, desta forma, elaborar estratégias de prevenção e de tratamento adaptadas a diferentes realidades.

Destarte, este trabalho visa contribuir com informações a todos aqueles que se interessam por essa temática. Ressalta-se a importância desse estudo para a sociedade como um todo, pois traz informações pautadas pela pesquisa capazes de alertar, orientar e conscientizar as pessoas sobre as consequências negativas relacionadas ao uso de substâncias psicoativas, em uma realidade atual na qual as estatísticas apontam para um crescente e elevado número de casos a cada novo levantamento. Assim, este artigo tem o objetivo de caracterizar as consequências do uso abusivo de substâncias psicoativas para pessoas em tratamento.

\section{METODOLOGIA}

Trata-se de uma pesquisa quantitativa, transversal e analítica, realizada no ano de 2012 em um Centro de Atenção Psicossocial Álcool e Drogas (CAPS AD), situado na região metropolitana de Curitiba. Dentre as 192 pessoas que estavam em tratamento no referido CAPS AD, 163 participaram da pesquisa. Os critérios de inclusão foram: ter idade de 18 anos ou mais e estar inserido em ao menos um grupo terapêutico. Foi excluído da amostra quem não possuía condições de ser entrevistado por falta de comunicação verbal (1) ou por estar sob o efeito de alguma substância psicoativa em todas as oportunidades de contato (1). Três participantes não foram informados sobre a pesquisa no momento da inserção no grupo terapêutico e, logo após, desistiram do tratamento não sendo possível o contato e 24 que não aceitaram participar da 
pesquisa após cinco tentativas de convite. A coleta de dados ocorreu por meio de entrevista estruturada, em sala reservada, após a autorização da coordenação do serviço, no período de janeiro a junho de 2012. Os participantes eram convidados a fazer parte da pesquisa no momento na assembleia geral que acontecia diariamente. Para aqueles que iniciavam o tratamento, o convite era realizado nos grupos de acolhimento.

Os dados foram coletados com instrumento que continham variáveis que permitiam identificar dados da caracterização do participante, do uso de substâncias psicoativas e das consequências acadêmicas, laborais, pessoais e familiares. Tal instrumento foi elaborado com base na literatura e com o auxílio de um profissional de estatística.

Utilizou-se a analise descritiva referente à caracterização sociodemográfica, do uso e do impacto do uso de substâncias psicoativas considerando os aspectos laborais e acadêmicos, pessoais e familiares. Foi realizada a análise da existência ou não de significância entre as variáveis de interesse dentro de cada grupo, para tanto se aplicou o teste de correlação de Pearson, em nível de significância de $p \leq 0,05$. Os softwares utilizados na análise dos dados foram o Microsoft Excel ${ }^{\circledR} 2010$ for Windows e o SPSS ${ }^{\circledR} 19.0$.

Esta pesquisa faz parte do projeto de pesquisa intitulado: Perfil dos dependentes químicos em um Centro de Atenção Psicossocial Álcool e Outras Drogas aprovado pelo Comitê de Ética em Pesquisa do Setor de Ciências da Saúde da Universidade Federal do Paraná, sob a inscrição CAAE: 0184.0.091.000-11.

\section{RESULTADOS}

Os resultados apresentados são referentes somente ao sexo masculino pelo baixo quantitativo do sexo feminino $(n=13)$ impossibilitando de chegar a resultado fidedigno. Em relação à caracterização dos participantes, identificou-se que a média etária foi de 41 anos, com desvio- padrão (Dp) de \pm 11 . O ensino fundamental incompleto foi encontrado em $58,7 \%(n=88), 46 \%$ eram solteiros $(n=69), 45,3 \%(n=68)$ estavam desempregados e $50 \%(n=75)$ possuíam renda familiar até três salários mínimos.
Identificou-se que a média de idade de início de uso de substâncias psicoativas foi de 13,52 anos, com desvio-padrão de $\pm 3,8$, sendo o álcool a substância de primeiro contato em $74,7 \%(n=109)$, e avançou na vida adulta como a substância de preferência em 62,3\% dos participantes $(n=99)$, seguido pelo crack em $19,5 \%$ $(n=31)$. A frequência de uso foi diária em $95,3 \%$ dos participantes ( $n=143)$, sendo que $79,7 \%(n=114)$ faziam o uso quatro vezes ao dia ou mais.

Na Tabela 1 identifica-se correção positiva entre substância de preferência e a idade atual, ou seja, aqueles que utilizam álcool como substância de preferência, possuem a média de idade entre 44,5 anos com desvio-padrão de 9,3 e aqueles que utilizam o crack, possuem uma média de idade inferior de 35,1 anos com desvio-padrão de 10,7 anos.

Tabela 1. Impacto do uso de substâncias psicoativas considerando aspectos sociodemográficos. Colombo, PR, Brasil, 2012. $(\mathrm{N}=150)$

(Continua)

\begin{tabular}{cccc}
\hline Variável & n & $\%$ & \multicolumn{2}{c}{$\begin{array}{c}\text { Pearson Correlation } p \\
\leq 0,05\end{array}$} \\
\hline Idade de inicio de Uso & & $\begin{array}{r}\text { Idade } \\
\text { atual }\end{array}$ & $\begin{array}{c}\text { Situação } \\
\text { Empregatícia }\end{array}$ \\
\hline
\end{tabular}

\begin{tabular}{|c|c|c|c|c|}
\hline$\leq$ que 17 anos & 134 & 89,3 & & \\
\hline $18 \mid---19$ anos & 9 & 6 &, 052 & ,152 \\
\hline $20 \mid--29$ anos & 7 & 4,7 & & \\
\hline $30 \mid---39$ anos & 0 & 0 & & \\
\hline Total & 150 & 100 & & \\
\hline
\end{tabular}

\begin{tabular}{|c|c|c|c|c|}
\hline Frequência d & & & & \\
\hline Diariamente & 143 & 95,3 & & \\
\hline $\begin{array}{l}\text { Três vezes na } \\
\text { semana }\end{array}$ & 5 & 3,3 & ,324 & ,476, \\
\hline $\begin{array}{l}\text { Duas vezes na } \\
\text { semana }\end{array}$ & 2 & 1,3 & & \\
\hline Total & 150 & 100 & & \\
\hline $\begin{array}{l}\text { Substancia d } \\
\text { Preferência* }\end{array}$ & & & & \\
\hline Álcool & 99 & 62,3 & & \\
\hline Crack & 31 & 19,5 & & \\
\hline
\end{tabular}


(Conclusão)

\begin{tabular}{lcccc}
\hline \multicolumn{1}{c}{ Variável } & $\mathbf{n}$ & $\%$ & $\begin{array}{c}\text { Pearson Correlation } p \\
\leq 0,05\end{array}$ \\
\hline Cocaína & 15 & 9,4 &, 000 &, 839 \\
Tabaco & 7 & 4,4 & & \\
Maconha & 5 & 3,1 & \\
Medicamentos & 1 & 0,6 & \\
Inalantes & 1 & 0,6 & \\
Total & 159 & 100 & \\
\hline
\end{tabular}

Na Tabela 2 são apresentados dados do impacto do uso de substâncias psicoativas referente aos aspectos acadêmicos e laborais. Identificou-se significância estatística entre o abandono dos estudos e a idade e entre o benefício previdenciário e a situação empregatícia. Destaca-se que $73,7 \%$ daqueles que estavam com a carteira de trabalho assinada recebiam, em decorrência dos transtornos relacionados a substâncias, benefício financeiro previdenciário e $68,4 \%$ o auxílio-doença. Com relação ao abandono dos estudos e a idade, $42 \%$ que abandonaram os estudos possuíam uma média de idade de 43,2 anos com desvio-padrão de 10,081.

Tabela 2. Impacto do uso de substâncias psicoativas considerando aspectos acadêmicos e laborais. Colombo, PR, Brasil, 2012. $(\mathrm{N}=150)$

\begin{tabular}{lccccc}
\hline Variável & $\mathbf{n}$ & $\%$ & \multicolumn{4}{c}{ Pearson Correlation $p \leq 0,05$} \\
\hline \multicolumn{2}{c}{ Abandono dos estudos } & Idade & $\begin{array}{c}\text { Escolari- } \\
\text { dade }\end{array}$ & $\begin{array}{c}\text { Situação Em- } \\
\text { pregatícia }\end{array}$ \\
\hline Não & 87 & 58 & & & \\
Sim & 63 & 42 &, 002 &, 200 & 0,86 \\
Total & 150 & 100 & & & \\
\hline Problemas no trabalho & & & \\
\hline Sim & 95 & 63,3 & .401 & .220 & \\
Não & 55 & 36,7 & & & \\
Total & 150 & 100 & & & \\
\hline Benefício previdenciário & & & \\
\hline Sim & 57 & 38 &, 303 &, 334 & \\
Não & 93 & 62 & & & \\
Total & 150 & 100 & & & \\
\hline
\end{tabular}

Na Tabela 3, apresentam-se dados do impacto pessoal do uso de substâncias psicoativas. Foram analisadas as variáveis referentes à overdose, comorbidades físicas e mentais, tentativa de suicídio, DST's e consequências criminais.

Pelo uso de substâncias psicoativas, 34,7\% tiveram episódios de overdose acidental sendo que $98 \%$ utilizavam substâncias psicoativas diariamente, $66 \%(n=99)$ apresentaram comorbidades físicas e $58,7 \% \quad(n=88)$ comorbidades mentais. Dentre os participantes, 60\% $(n=90)$ tiveram ideação suicida e 30,7\% $(n=46)$ chegaram a tentar o suicídio, com destaque para aqueles que usam álcool em $50 \%(n=23)$ dos casos com frequência diária.

Em relação ao comportamento sexual de risco, $13,3 \%(n=20)$ fizeram sexo em troca de dinheiro para comprar as substâncias psicoativas e 12,7\% $(n=19)$ em troca dessas. Dentre os participantes, 32,7\% $(n=49)$ já desenvolveram alguma DST's. Relativo à criminalidade, $40,7 \%(n=61)$ se evolveram com algum delito.

Tabela 3. Impacto do uso de substâncias psicoativas considerando aspectos pessoais. Colombo, PR, Brasil, 2012. $(\mathrm{N}=150)$

(Continua)

\begin{tabular}{lccccc}
\hline \multicolumn{1}{c}{ Variável } & $\mathbf{n}$ & $\%$ & \multicolumn{3}{c}{ Pearson Correlation $p \leq 0,05$} \\
\hline \multicolumn{1}{c}{ Overdose } & & & $\begin{array}{c}\text { Idade de } \\
\text { início }\end{array}$ & $\begin{array}{c}\text { Substân- } \\
\text { cia }\end{array}$ & $\begin{array}{c}\text { Frequência } \\
\text { de uso }\end{array}$ \\
2 vezes ou mais & 26 & 50 & & .446 & .014 \\
1 vez & 24 & 46,2 & .411 & & \\
$\begin{array}{l}\text { Não sabe infor- } \\
\text { mar }\end{array}$ & 2 & 3,8 & & & \\
Total & 52 & 100 & & & \\
\hline $\begin{array}{c}\text { Comorbidades } \\
\text { físicas }\end{array}$ & & & $\begin{array}{c}\text { Idade } \\
\text { atual }\end{array}$ & $\begin{array}{c}\text { Idade de } \\
\text { início }\end{array}$ & $\begin{array}{c}\text { Frequên- } \\
\text { cia de } \\
\text { uso }\end{array}$
\end{tabular}

\begin{tabular}{lccccc}
$\begin{array}{l}\text { Sistema Car- } \\
\text { diovarcular }\end{array}$ & 51 & 27,4 & & & \\
$\begin{array}{l}\text { Sistema Neu- } \\
\text { rológico }\end{array}$ & 46 & 24,7 & & \\
$\begin{array}{l}\text { Sistema Gas- } \\
\text { tro Intestinal }\end{array}$ & 31 & 16,7 & .000 & .403 &, 051 \\
$\begin{array}{l}\text { Sistema He- } \\
\text { pático }\end{array}$ & 25 & 13,4 & & \\
$\begin{array}{l}\text { Outros } \\
\text { Sistema En- } \\
\text { dócrino }\end{array}$ & 24 & 12,9 \\
Total & 186 & 100 \\
\hline
\end{tabular}


(Continua)

\begin{tabular}{|c|c|c|c|c|c|}
\hline Variável & $\mathbf{n}$ & $\%$ & \multicolumn{3}{|c|}{ Pearson Correlation $p \leq 0,05$} \\
\hline $\begin{array}{l}\text { Comorbida- } \\
\text { des mentais }\end{array}$ & & & $\begin{array}{l}\text { Substância } \\
\text { de prefe- } \\
\text { rência }\end{array}$ & $\begin{array}{l}\text { Idade de } \\
\text { início de } \\
\text { uso }\end{array}$ & $\begin{array}{l}\text { Frequência } \\
\text { de uso }\end{array}$ \\
\hline $\begin{array}{l}\text { Trantornos } \\
\text { psicóticos }\end{array}$ & 35 & 56,5 & & & \\
\hline $\begin{array}{l}\text { Trantornos } \\
\text { do humor }\end{array}$ & 23 & 37,1 & .228 & .051 & .209 \\
\hline $\begin{array}{l}\text { Trantornos } \\
\text { do comporta- } \\
\text { mento }\end{array}$ & 1 & 1,6 & & & \\
\hline Outros & 2 & 3,2 & & & \\
\hline $\begin{array}{l}\text { Não soube } \\
\text { responder }\end{array}$ & 1 & 1,6 & & & \\
\hline Total & 62 & 100 & & & \\
\hline $\begin{array}{l}\text { Tentativa de } \\
\text { suicídio }\end{array}$ & & & $\begin{array}{l}\text { Substân- } \\
\text { cia }\end{array}$ & $\begin{array}{l}\text { Idade de } \\
\text { início }\end{array}$ & $\begin{array}{l}\text { Frequên- } \\
\text { cia }\end{array}$ \\
\hline $\begin{array}{l}\text { Intoxicação } \\
\text { exógena }\end{array}$ & 18 & 26,5 & & & \\
\hline $\begin{array}{l}\text { Ferimento } \\
\text { perfurocor- } \\
\text { tante }\end{array}$ & 17 & 25 & & & \\
\hline $\begin{array}{l}\text { Enforcamen- } \\
\text { to }\end{array}$ & 10 & 14,7 & & & \\
\hline Atropelado & 7 & 10,3 & & & \\
\hline $\begin{array}{l}\text { Queda de } \\
\text { nível }\end{array}$ & 6 & 8,8 & .050 & .065 & .038 \\
\hline Afogado & 3 & 4,4 & & & \\
\hline Arma de fogo & 3 & 4,4 & & & \\
\hline Queimadura & 2 & 2,9 & & & \\
\hline Outros & 2 & 2,9 & & & \\
\hline Total & 68 & 0100 & & & \\
\hline
\end{tabular}

\begin{tabular}{|c|c|c|c|c|c|}
\hline Variável & n & $\%$ & \multicolumn{3}{|c|}{ Pearson Correlation $p \leq 0,05$} \\
\hline \multicolumn{6}{|c|}{ Agressão de Outrem } \\
\hline $\begin{array}{l}\text { Desconheci- } \\
\text { dos }\end{array}$ & 80 & 72,7 & & & \\
\hline Familiares & 21 & 19,1 & & & \\
\hline Amigos & 8 & 7,3 & & & \\
\hline Polícia & 1 & 0,9 & & & \\
\hline Total & 110 & 100 & & & \\
\hline $\begin{array}{l}\text { Consequên- } \\
\text { cias criminais }\end{array}$ & & & Substância & $\begin{array}{l}\text { Escolari- } \\
\text { dade }\end{array}$ & $\begin{array}{c}\text { Situação } \\
\text { emprega- } \\
\text { tícia }\end{array}$ \\
\hline Roubo & 14 & 23 & & & \\
\hline Outros & 9 & 14,8 & & & \\
\hline Agressão & 13 & 21,3 & & & \\
\hline $\begin{array}{l}\text { Porte de } \\
\text { drogas }\end{array}$ & 6 & 9,8 & & & \\
\hline $\begin{array}{l}\text { Tráfico de } \\
\text { drogas }\end{array}$ & 6 & 9,8 & .039 & .006 & .039 \\
\hline Assalto & 5 & 8,2 & & & \\
\hline $\begin{array}{l}\text { Tentativa de } \\
\text { Homicídio/ } \\
\text { Homicídio }\end{array}$ & 4 & 6,6 & & & \\
\hline Porte de arma & 2 & 3,3 & & & \\
\hline Estelionato & 2 & 3,3 & & & \\
\hline Total & 61 & 100 & & & \\
\hline
\end{tabular}

O impacto do uso de drogas também foi identificado por meio das consequências familiares e, dentre os participantes, $70 \%(\mathrm{n}=105)$ tinham histórico familiar de uso de substâncias psicoativas, $46 \%(n=69)$ tiveram rompimento de vínculos e $70 \%(\mathrm{n}=105)$ problemas conjugais. Dentre aqueles que tiveram rompimento de vínculos, 37,3\% e desses, $40 \%$ já estiveram em situação de rua.

$\begin{array}{lll}\text { Gonorréia } & 15 & 57,7 \\ \text { Síflis } & 3 & 11,5 \\ \text { HIV } & 3 & 11,5 \\ \text { Herpes } & 3 & 11,5 \\ \text { Cancro Mole } & 2 & 7,7 \\ \text { Total } & 26 & 100\end{array}$

de risco

Tabela 4. Impacto do uso de substâncias psicoativas considerando aspectos familiares. Colombo, PR, Brasil, 2012. $(\mathrm{N}=150)$

(Continua)

Agressão Física

\begin{tabular}{lcl}
\hline $\begin{array}{l}\text { Desconheci- } \\
\text { dos }\end{array}$ & 79 & 60,8 \\
Familiares & 39 & 30 \\
Amigos & 12 & 9,2 \\
Total & 130 & 100 \\
\hline
\end{tabular}

\begin{tabular}{lcccc}
\hline \multicolumn{1}{c}{ Variável } & n & $\%$ & Pearson Correlation $p \leq 0,05$ \\
\hline $\begin{array}{c}\text { Histórico fami- } \\
\text { liar }\end{array}$ & & & $\begin{array}{c}\text { Substância de } \\
\text { preferencia }\end{array}$ & $\begin{array}{c}\text { Rompi- } \\
\text { mento de } \\
\text { vínculo }\end{array}$ \\
Outros & 101 & 44,7 & & \\
Irmãos & 90 & 39,8 & &, 207 \\
Pai / Mãe & 27 & 11,9 &, 161 & \\
Filhos & 7 & 3,1 & & \\
\hline
\end{tabular}


(Conclusão) pesquisa é corroborado aos dados de outros estudos, na-

\begin{tabular}{|c|c|c|c|}
\hline Variável & $\mathbf{n}$ & $\%$ & Pearson Correlation $p \leq 0,05$ \\
\hline Cônjuge & 1 & 0,4 & \\
\hline Total & 226 & 100 & \\
\hline \multicolumn{4}{|c|}{$\begin{array}{l}\text { Substância de } \\
\text { abuso* }\end{array}$} \\
\hline Álcool & 153 & 67,7 & \\
\hline $\begin{array}{l}\text { Múltiplas dro- } \\
\text { gas }\end{array}$ & 38 & 16,8 & ,353 \\
\hline Crack & 21 & 9,3 & ,111 \\
\hline Cocaína & 8 & 3,5 & \\
\hline Maconha & 6 & 2,7 & \\
\hline Total & 226 & 100 & \\
\hline
\end{tabular}

\begin{tabular}{lclll}
\hline $\begin{array}{l}\text { Rompimento de } \\
\text { vínculos }\end{array}$ & & & & \\
Conjuge & 13 & 21,7 & & \\
Irmãos & 7 & 11,7 & &, 321 \\
Outros & 3 & 5 &, 353 & \\
Sogra & 10 & 16,7 & & \\
Filhos & 13 & 21,7 & & \\
Mãe/Pai & 14 & 23,3 & & \\
Total & 60 & 100 & & \\
\hline
\end{tabular}

\begin{tabular}{lcccc}
\hline $\begin{array}{l}\text { Problemas Con- } \\
\text { jugais }\end{array}$ & & & & \\
Discussões & 48 & 45,7 & & \\
Outros & 27 & 25,7 & & \\
$\begin{array}{l}\text { Não quiseram } \\
\text { responder }\end{array}$ & 19 & 18,1 & &, 231 \\
Agressões & 7 & 6,7 &, 134 & \\
$\begin{array}{l}\text { Disputa por } \\
\text { drogas }\end{array}$ & 4 & 3,8 & & \\
Total & 105 & 100 & & \\
\hline
\end{tabular}

\section{Abandono do lar}

\begin{tabular}{lcccc} 
Não & 94 & 62,7 & & \\
Sim & 56 & 37,3 &, 139 &, 001 \\
Total & 150 & 100 & & \\
\hline
\end{tabular}

\section{Situação de Rua}

\begin{tabular}{lcccc} 
Não & 90 & 60 & & \\
Sim & 60 & 40 &, 207 &, 901 \\
Total & 150 & 100 & & \\
\hline
\end{tabular}

\section{DISCUSSÃO}

O elevado quantitativo de homens com transtornos relacionados a substâncias em relação às mulheres com esse mesmo transtorno mental encontrado nesta

cionais e internacionais $^{8.9}$. Cabe considerar que as informações que compõem tais estudos são relativas somente a dados de instituição, uma população restrita que procurou tratamento, o que não reflete o real quantitativo de mulheres que usam substâncias psicoativas que estão distribuídas na sociedade, que pode ser muito maior.

A idade dos participantes da presente pesquisa com predominância na faixa etária de 30 a 49 anos com média de 41 anos são semelhantes a um estudo documental realizado com 350 prontuários de dependente químico em tratamento integral no Estado do Paraná, onde a média de idade foi de 35,8 anos, com desvio-padrão de $11,9^{10}$. Normalmente, na vida adulta, os usuários já tiveram muito sofrimento em decorrência dos transtornos relacionados a substâncias e, portanto, desenvolvem insight para a mudança de comportamento. Contudo, apesar do intenso sofrimento e dos constantes prejuízos, é possível buscar melhoria na qualidade de vida e resgatar possíveis perdas se tiver um tratamento adequado nessa faixa etária ${ }^{10-11}$.

O estado civil solteiro foi encontrado em outras pesquisas com a mesma característica da população do presente estudo 2,9,12. O fato de grande parte dos estudos com pessoas com transtornos relacionados a substâncias apresentarem essa característica é atribuído à dificuldade em manter-se em relacionamento familiar afetivo, em decorrência do seu intenso envolvimento com a droga.

O ensino fundamental incompleto foi o nível escolar predominante na amostra desta pesquisa com uma média de idade de 38 anos, idade produtiva do indivíduo, no qual se espera que ao menos tenha finalizado o período acadêmico, fato referido por apenas $12 \%$ dos participantes. A baixa escolaridade se assemelha aos dados escolares de vários estudos ${ }^{10,13}$, nos quais apontaram um percentual acima de $60 \%$ pessoas com transtornos relacionados a substâncias que possuíam o ensino fundamental. Isso é uma questão preocupante à medida que este quadro reflete em uma baixa qualificação profissio$\mathrm{nal}^{10}$.

Constata-se que entre os participantes desta pesquisa, grande parte estava desempregada e tiveram múltiplos problemas no ambiente de trabalho em conse- 
quência do uso de drogas e, por fim, necessitou mudar de atividade. $\mathrm{O}$ uso de substâncias psicoativas no meio corporativo é considerado muito destrutivo, pois gera perdas no patrimônio das empresas, adoecimento, afastamento e baixa produtividade, a pessoa com transtornos relacionados a substâncias utiliza somente em torno de $67 \%$ de sua capacidade de trabalho ${ }^{14}$.

Esse baixo rendimento ocorre geralmente porque os funcionários vão ao trabalho sob o efeito de alguma substância química. Desta forma estão mais propensos a cometer erros no momento do trabalho, especialmente porque a droga altera a concentração, dificulta a atenção, facilita a perda de reflexos e altera a cogniçãa ${ }^{14}$.

A falta de escolaridade, a baixa qualificação profissional e o elevado quantitativo de desempregados podem ter refletido na baixa renda familiar dos participantes deste estudo que foi apontada entre um a três salários mínimos. Apesar de os transtornos relacionados à substância atingir diferentes grupos sociais, pessoas com ou sem instrução, jovens ou adultos, a classe social menos favorecida, é considerada um dos principais fatores de risco no desenvolvimento desse transtorno mental ${ }^{1,14}$.

Identificou-se nesta pesquisa que o início do uso ocorreu na adolescência, etapa da vida considerada entre a infância e a fase adulta, reconhecida como um período crítico e complexo do processo de desenvolvimento biopsicossocial do indivíduo. ${ }^{1}$ Esse retrato quantitativo foi encontrado em outras pesquisas ${ }^{10,15}$, nas quais a idade média de início do uso foi de 14 anos para substâncias lícitas e de 19 anos para as ilícitas.

Pode-se perceber que o hábito de utilizar álcool perdurou após a adolescência, adentrando pela vida adulta o que resultou na dependência de $62,3 \%$ dos participantes da presente pesquisa por esta mesma substância, seguido por dependência do crack. Estudos ${ }^{9 \cdot 10}$ assinalam que aqueles que buscaram tratamento para os transtornos relacionados à substância foi em decorrência do álcool seguido pelo uso de outras substâncias, entre elas o crack.

Considerando a idade de início do uso de drogas e a idade média atual dos participantes, considera-se que o uso de drogas perdurou em torno de 27,4 anos. Pesqui$\mathrm{sa}^{16}$ destaca o tempo de uso de álcool elevado, de 22,3 anos para aqueles com idade até 59 anos e de 40,9 anos para aqueles com idade superior a 60 anos.
Apesar dos diferentes períodos de tempo de uso de drogas entre os diferentes estudos, percebe-que este hábito perdura por alguns anos e, nesse sentido, sabe-se que o uso prolongado de drogas agrava o prognóstico, pois ocorre a gravidade do transtorno o que compromete mais as atividades bem com as funções biopsicossociais do indivíduo ${ }^{16}$.

O uso de mais de uma substância encontrado nesta pesquisa e é sabido que se trata de uma prática comum, apesar de possuir uma substância de preferência, outras drogas são utilizadas por trazer efeito similar, ou minimizar o desconforto ocasionado pela síndrome de abstinência e como consequência amenizar a fissura ${ }^{10}$.

O uso de múltiplas drogas pode prejudicar o tratamento, pois a interação entre as drogas utilizadas de forma simultânea pode gerar efeitos clínicos, comportamentos adversos que dificultam a abstinência ou até mesmo pode levar ao uso da droga de preferência ${ }^{15}$.

A frequência de uso de drogas diária obteve a maior prevalência; quase que a totalidade faz o uso da droga de preferência todos os dias. Em pesquisa ${ }^{10}$ que visou o perfil do dependente químico em tratamento em um hospital psiquiátrico da região metropolitana de Curitiba, apontou que antes de iniciar o tratamento, 99,4\% de um total de 350 indivíduos, faziam uso diário de substâncias psicoativas.

No impacto pessoal foi identificada a ocorrência de overdose acidental por substâncias psicoativas. Embora, o uso de drogas esteja associado a diversas doenças físicas e mentais, é percebida há tempo sua forte relação ao risco de mortalidade precoce por overdose. Trata-se da principal causa de morte entre os usuários, sobretudo as acidentais, aquela que ocorre sem a intensão prévia do usuário $^{17}$.

As comorbidades mentais apresentadas por 41,3\% dos participantes desta pesquisa, dentre esses se destacaram os transtornos mentais com psicose e os de humor sem psicose. A relação entre os transtornos relacionados a substâncias e as comorbidades mentais vem sendo muito estudada na última década. Entretanto, apesar do aumento do número de estudos, pouco se sabe sobre essa correlação ${ }^{16}$. Compreende-se com certeza é que a associação entre dois ou mais transtornos mentais, seja de base ou de evolução posterior ao início do uso ${ }^{18}$. 
Quanto à tentativa de suicídio, sabe-se que a cada 40 segundos uma pessoa comete suicídio no mundo, sempre considerando que o número de tentativa supera o número de suicídio em pelo menos dez vezes. A idade de maior prevalência gira em torno de 15 a 35 anos e está entre as três maiores causas de morte e a sexta quando se trata de incapacitaçã $0^{16}$.

Quanto ao álcool ter sido a droga mais frequente entre os que tentaram suicídio, pode estar relacionado ao fato de que é a substância mais consumida em todo o mundo e a maior causa de dependência entre os participantes do presente estudo ${ }^{16}$. Dentre os que tentaram suicídio, houve destaque ao modo realizado, nesse caso a intoxicação exógena. Esse meio de tentativa de suicídio se enquadra entre os três principais modos utilizados, considerando o primeiro enforcamento, seguido pela arma de fogo. A escolha pela intoxicação exógena inclui aspectos psicossociais, de gênero, a disponibilidade de acesso e aceitabilidade sociocultural, pois se trata de modo pouco agressivo ${ }^{19}$.

Outro dado preocupante que emergiu da presente pesquisa foi em relação ao comportamento sexual dos participantes. É importante destacar que um comportamento sexual com riscos pode levar a inúmeros prejuízos quando associado a um transtorno mental por uso de substância, entre esses prejuízos destacam-se as DST's ${ }^{13}$.

Estudo ${ }^{13}$ destacou que o comportamento sexual de risco de seus participantes estava relacionado ao fato de que em muitos casos tiveram relações sexuais sob efeito de alguma substância psicoativa e fizeram trocas de favores sexuais como meio de conseguir sustentar a dependência. Essa associação merece atenção especial, porque em geral o usuário de substâncias psicoativas possui baixa adesão ao uso de preservativos, possui um início precoce da vida sexual e submente a encontrar-se com múltiplos parceiros ${ }^{13}$.

O comportamento agressivo e o envolvimento com a criminalidade estão diretamente relacionados ao uso de drogas, gerando um impacto importante para o indivíduo e para a sociedade ${ }^{21}$. Pode-se identificar esse perfil na presente pesquisa. $\mathrm{O}$ aumento progressivo da criminalidade, normalmente associado à falta de assistência familiar, omissão do governo e a carência de políticas públicas que visem ações sociais e assistências ${ }^{20}$.
De acordo com estudo ${ }^{21}$ dentre os principais fatores atribuídos à criminalidade relacionado ao uso de substâncias psicoativas, estão a influência dos antecedentes familiares do indivíduo, abuso físico, negligência, experiências inadequadas de socialização e agressões durante a infância ou na adolescência. Quando relacionado a histórico de comorbidades mentais, o índice se eleva. Os antecedentes culturais como valores adquiridos, crenças e normas internalizadas impostas pela família e pela sociedade são fatores importantes relacionados à criminalidade e à pessoa com transtornos relacionados a substâncias, pois podem evitar a prática de um delito ou favorecer para que ela aconteça ${ }^{20}$.

O impacto familiar do uso de drogas foi percebido por meio do histórico familiar de consumo. Pesquisas apontam o elevado índice de familiares que também apresentam transtornos relacionados a substâncias e destacam um quantitativo acima de 60\%. O álcool emergiu como a substância mais utilizada pelos familiares na presente pesquisa. O histórico de alcoolismo na família predispõe o desenvolvimento desse mesmo problema entre outros membros. Este fato pode ser iniciado por questões genéticas ou por influência comportamental ${ }^{10,12}$. Entende-se que filhos de pessoas com transtornos relacionados a substâncias apresentam um risco aumentado para o consumo problemático de álcool e outras drogas, quando comparados a filhos de pessoas que não usam $\operatorname{drogas}^{21}$.

Em relação ao rompimento de vínculo com os familiares, entende-se que a convivência de familiares com usuários de drogas é afetada com a progressão do uso causando inúmeros sofrimentos e sentimentos de resignação, solidão, frustação e tristeza o que compromete os vínculos e implica em seu rompimento ${ }^{21}$.

A comunicação é prejudicada em famílias disfuncionais, pois as drogas geram funcionamento patológico, caracterizado pela ausência de expressão de sentimentos, ideias ou opiniões, bem como a maneira de se falar e ser ouvido. O divórcio ocorre porque na relação conjugal não há mais diálogo, responsabilidades, há falta de cooperação, insatisfação marital, desejo sexual diminuído, ciúmes, estresse e violência ${ }^{21}$.

Em muitos casos os problemas de relacionamento familiar, relativo ao uso da droga, leva o indivíduo a 
sair de casa e sem ter onde ficar acaba por permanecer em situação de rua. Estar em situação de rua pode estar associado a diversas situações como o desemprego, falta de moradia e outros contextos sociais e econômicos desfavoráveis, no entanto, é comum identificar pessoas em situação de rua em decorrência de rompimento de vínculos familiares ${ }^{22}$.

\section{CONCLUSÃO}

Conclui-se que foi possível identificar o impacto do uso de drogas pelo dependente químico. Os participantes desta pesquisa, em sua maioria, são homens, adultos, solteiros, com o ensino fundamental incompleto, desempregados e baixa renda, características comuns entre usuários de drogas que pode ser tanto consequência como fatores de risco para o consumo. Chama a atenção o início do uso de drogas na adolescência, bem como para o tipo de droga de início, o álcool e o tabaco.

Em geral, não há reflexão crítica por parte da população acerca dos problemas que envolvem o uso de drogas precocemente e de certa maneira há negligência social que permite o contato por meio de confraternizações, festas familiares, comerciais, publicidades entre outros. Esse fato pode ser uma consequência da escassa preocupação do governo com esse grave problema de saúde pública, que parece se abster ou pouco fazer para dificultar o acesso de adolescentes à venda e ao consumo dessas substâncias.

Destaca-se o número de mulheres como participantes do presente estudo que não foi abordada na discussão do estudo em decorrência do baixo quantitativo e, por consequência, a impossibilidade de realizar testes estatísticos fidedignos que apresentasse a realidade local. Mas, sabe-se que há crescente número de usuárias na atualidade camuflada muitas vezes pela vergonha e pelo preconceito social que envolve toda questão da posição que exerce na sociedade que dificulta a procura por tratamento.

O fato de esta pesquisa ser realizada em um único município brasileiro, impossibilita a generalização dos dados. No entanto, todos os resultados vão ao encontro de outros estudos nacionais e internacionais sobre as consequências do uso de drogas o que denota a importância dos dados que emergiram no presente estudo.
Destarte, entende-se que o desenvolvimento de estratégias de educação e prevenção é a uma questão que deve ser analisada e aprofundada pelos serviços públicos, pois pode ser extremamente útil para minimizar o impacto que o uso abusivo de drogas gera.

\section{REFERÊNCIAS}

1. Diehl A, Cordeiro DC, Laranjeira R. Dependência química: prevenção, tratamento e políticas públicas. Porto Alegre: Artmed; 2011.

2. Lle S, Hsu J, Tsay W. The trend of drug abuse in Taiwan during the years 1990 to 2011. J Food Drug Anal. 2013; 21(4):390-6.

3. Manual diagnóstico e estatístico de transtornos mentais. American Psychiatric Association. 5 ed. Porto Alegre, RS: Artmed; 2014.

4. World Health Organization - WHO. Substance Abuse, 2013. [Internet]. [Acesso em 23 maio 2013]. Disponível em: http://www.who.int/topics/substance_abuse/ en/

5. United Nations Oficce of Drugs and Crime (UNODC). World drug report. [internet] United Nations publication, 2017. [Acesso em: 31 jan. 2018]. Disponível em: http://www.unodc.org/documents/data-and-analysis/ WDR2012/WDR_2012_web_small.pdf.

6. Carlini EA, Noto AR, Sanchez ZM, Carlini CMA, Locatelli DP, Abeid LR, et al. VI Levantamento Nacional sobre o Consumo de Drogas Psicotrópicas entre Estudantes do Ensino Fundamental e Médio das Redes Pública e Privada de Ensino nas 27 Capitais Brasileiras -2010. Brasília, DF: SENAD; 2010.

7. Grigsby TJ, Forster M, Baezconde-Garbanati L, Soto DW, Unger JB. Do adolescent drug user consequences predict externalizing and internalizing problems in emerging adulthood as well as traditional drug use measures in a Hispanic sample? Addict Behav. 2014; 39(3): 644-51.

8. Freire SD, Santos PL, Bortolini, M, Moraes JFD, Oliveira MS. Intensidade de uso de crack de acordo com a classe econômica de usuários internados na cidade de Porto Alegre/Brasil. J Bras Psiquiatr. [internet] 2012; 61(4):221-6. [Acesso em 23 maio 2013]. 
Disponível em: http://dx.doi.org/10.1590/S004720852012000400005.

9. Almeida RA, Anjos UU, Viana RPT, Pequeno GA. Perfil dos usuários de substâncias psicoativas de João Pessoa. Saúde Debate. 2014; 38(102):526-38. [Acesso em 23 maio 2013]. Disponível em: http://www.scielo.br/ pdf/sdeb/v38n102/0103-1104-sdeb-38-102-0526.pdf

10. Capistrano FC, Ferreira ACZ, Silva TL, Kalinke LP, Maftum MA. Perfil sociodemográfico e clínico de dependentes químicos em tratamento: análise de prontuários. Esc Anna Nery Rev Enferm. 2012; 17(2): 234-41. [Acesso em 23 maio 2013]. Disponível em: http://dx. doi.org/10.1590/S1414-81452013000200005.

11. Silva, CR, Kolling NM, Carvalho JCN, Cunha SM, Kristensen $\mathrm{CH}$. Comorbidade psiquiátrica em dependentes de cocaína/crack e alcoolistas: um estudo exploratório. Aletheia. 2009; 30:101-12. [Acesso em 23 maio 2013]. Disponível em: http:// pepsic.bvsalud.org/scielo.php?script =sci_arttext\&pi$\mathrm{d}=$ S1413-03942009000200009.

12. Ferreira ACZ, Capistrano FC, Maftum MA, Kalinke LP, Kirchhof ALC. Caracterização de internações de dependentes químicos em uma unidade de reabilitação. Cogitare Enferm. 2012; 17(3):444-51. [Acesso em 23 maio 2013]. Disponível em: http://ojs.c3sl.ufpr.br/ojs/ index.php/cogitare/article/viewFile/29284/19033.

13. Machado CA, Moreira T, Ferigolo M, Oliveira M, Barros HMT. Envolvimento de clientes com diferentes formas de violência em um serviço de 133 teleatendimento para dependência química. Rev. Saúde Públ Santa Cat. 2012; 5(2):22-9. [Acesso em 23 maio 2013]. Disponível em: http://esp.saude.sc.gov.br/sistemas/revista/index.php/inicio/article/viewFile/135/165.

14. Spezzia S, Vicente SP, Calvoso Júnior R. Acidentes de trabalho causados pelo uso de drogas. UNINGÁ Review. 2013; 14(1):5-12. [Acesso em 23 maio 2013]. Disponível em: http://www.mastereditora.com.br/periodico/20130701_165638.pdf.

15.Zeni TC, Araújo RB. Relação entre o craving por tabaco e o craving por crack em pacientes internados para desintoxicação. J Bras Psiquiatr. 2011; 60(1):2833. [Acesso em 23 maio 2013]. Disponível em: http:/ www.scielo.br/scielo.php?script =sci_arttext\&pi$\mathrm{d}=$ S0047-20852011000100006.
16.Zupanc T, Agius M, Paska AV, Pregelj P. Bood alcohol concentration of suicide victims by partial hanging. J Forensic Leg Med. 2013; 20(8):976-9.

17. Bartoli F, Carrà G, Brambilla G, Carreta D, Crocamo $\mathrm{C}$, Neufeind J, et al. Association between depression and non-fatal overdoses among drug users: a systematic review and meta-analysis. Drug Alcohol Depend. 2014; 134:12-21.

18. Hess ARB, Almeida RMM, Moraes AL. Comorbidades psiquiátricas em dependentes químicos em abstinência em ambiente protegido. Estud Psicol. 2012; 17(1):171-9. [Acesso em 23 maio 2013]. Disponível em: http://www.scielo.br/scielo.php?script $=$ sci_arttext\&pid $=$ S1413-294X2012000100021.

19. Santos AS, Legay LF, Lovisi GM. Substâncias tóxicas e tentativas de suicídios: considerações sobre acesso e medidas restritivas. Cad. Saúde Colet. 2013; 21(1):5361. [Acesso em 23 maio 2013]. Disponível em: http:// www.scielo.br/scielo.php?script =sci_arttext\&pi$\mathrm{d}=$ S1414-462X2013000100009.

20. Cortez FCP, Baltieri DA. Consumo nocivo de álcool e violência. Rev Criminol Ciênc Penitenciárias. 2011; 1(2):1-38. [Acesso em 23 maio 2013]. Disponível em: http://www.procrim.org/revista/index.php/COPEN/article/view/26.

21. Mangueira SO, Lopes MVO. Família disfuncional no contexto do alcoolismo: análise de conceito. Rev Bras Enferm. 2014; 67(1):149-54. [Acesso em 23 maio 2013]. Disponível em: http://www.scielo.br/scielo.php?scrip$\mathrm{t}=$ sci_arttext\&pid=S0034-71672014000100149.

22. Farias DCS, Rodrigues ILA, Marinho IC, Nogueira LMV. Saberes sobre saúde entre pessoas vivendo em situação de rua. Psicologia e Saber Social. [Internet] 2014; 3(1):70-82. [Acesso em 23 maio 2013]. Disponível em: http://www.e-publicacoes.uerj.br/index.php/ psi-sabersocial/article/view/10064/9504.

Recebido em: 08/07/2017 Aceito em: 22/02/2018 